\title{
A STUDY ON AETIOLOGICAL AGENTS OF LRTI WITH SPECIAL EMPHASIS ON DRUG SENSITIVITY PATTERN OF ISOLATED BACTERIA AND INCIDENCE OF PNEUMOCYSTIS CARINII PNEUMONIA
}

\author{
${ }^{1}$ Assistant Professor, Department of Tropical Medicine, CSTM, Kolkata. \\ ${ }^{2}$ Professor, Department of Tropical Medicine, CSTM, Kolkata. \\ ${ }^{3}$ Assistant Professor, Department of Microbiology, CSTM, Kolkata. \\ ${ }^{4}$ Trainee, Department of Microbiology, CSTM, Kolkata. \\ 5 Medical Technologist, CSTM, Kolkata. \\ ${ }^{6}$ Medical Technologist, CSTM, Kolkata.
}

Manab Kumar Ghosh', Bibhuti Saha², Indrani Bhattacharyya ${ }^{3}$, Mandira Chakraborty ${ }^{4}$ Debabrata Roy5, Md. Samidul Hoque 6

ABSTRACT

Lower Respiratory Tract Infections (LRTIs) are important causes of morbidity and mortality for all age groups both in the hospital as well as in the community. Though international guidelines are available for treating bacterial LRTI they have not been validated very much in the Indian scenario. Scarce local information is available regarding aetiological agents of LRTI and their antibiotic sensitivity pattern. Pneumocystis carinii (Jiroveci) is a common pathogen in LRTI in immuno-compromised patients. It is rarely documented in our country.

\section{MATERIALS AND METHODS}

Two hundred adults suffering from LRTI of less than 2 weeks duration from both inpatient and outpatient departments in a Tertiary Care Hospital at Kolkata were included in the study after taking valid informed consent. Study period was one year (201415). Sputum samples were collected as per criteria of Murray et al. 1975. After performing routine culture, diagnosis was made based on colony character and biochemical reactions. Antimicrobial sensitivity testing was then done according to CLSI guidelines.

\section{RESULTS}

This study reveals that, LRTI was common in all age groups, in both Human Immunodeficiency Virus Non-Reactive (HIVNR) and Human Immunodeficiency Virus Reactive (HIVR) groups. In both the groups, $75 \%$ showed growth of single organism in sputum. But in the HIVR group, a considerable number showed growth of multiple organisms. Both the groups (HIVNR and HIVR) showed predominance of Gram positive bacteria. Antibiotic sensitivity was unremarkable, but one E.coli isolate was an Extended Spectrum Beta-Lactamase producer (ESBL). Pneumocystis Carinii (PC) was found in Giemsa stained smear of induced sputum in one HIVR case, who developed Pneumo-mediastinum and surgical emphysema, but recovered due to early initiation of treatment, suggesting that Pneumocystis Carinii (Jiroveci) pneumonia was not uncommon in HIVR patients and early diagnosis and treatment can save the life of the patient.

\section{CONCLUSION}

In our study LRTI was found to be equally common in both HIV-R and HIV-NR patients. Gram positive bacteria were the predominant pathogens in both groups. But double and multiple infection was found in the HIV-R cohort. Antibiotic sensitivity pattern was unremarkable. One E.coli isolate was an ESBL producer. Pneumocystis Jiroveci detection was done in induced sputum.

\section{KEYWORDS}

Lower respiratory tract infections, Pneumocystis carinii, HIV, Pneumonia, Antibiotic, Sputum.

HOW TO CITE THIS ARTICLE: Ghosh MK, Saha B, Bhattacharyya I, et al. A study on aetiological agents of LRTI with special emphasis on drug sensitivity pattern of isolated bacteria and incidence of pneumocystis carinii pneumonia. J Evolution Med Dent Sci 2016;5(1): 9-14, DOI: $10.14260 /$ jemds $/ 2016 / 3$

\section{INTRODUCTION}

Lower Respiratory Tract Infections (LRTIs) are among the most common infectious diseases affecting human beings worldwide. ${ }^{1}$ they are important causes of morbidity and mortality for all age groups both in the hospital as well as in the community. Each year approximately 7 million people die as a direct consequence of acute and chronic respiratory infections..$^{2}$ Age, gender and season are factors that have been implicated to affect the prevalence of LRTIs. ${ }^{3}$

Financial or Other, Competing Interest: None.

Submission 25-12-2015, Peer Review 26-12-2015,

Acceptance 30-12-2015, Published 01-01-2016.

Corresponding Author:

Dr. Indrani Bhattacharyya,

$37 \mathrm{~J} / 1$, Raja Manindra Road,

Kolkata-700037.

E-mail: indranichaudhuri@yahoo.com

DOI:10.14260/jemds/2016/3
The aetiological agents of LRTIs vary from area to area. ${ }^{2,4}$ so the susceptibility profile will also differ between geographical locations.

Malaria is caused by four species of the genus Plasmodium, namely Plasmodium vivax, Plasmodium falciparum, Plasmodium malariae and P. ovale. As per World Health Organisation, the major manifestations of severe malaria are cerebral malaria, severe anemia, jaundice, Acute Renal Failure (ARF), Acute Respiratory Distress Syndrome (ARDS), shock, disseminated major bacterial pathogens being isolated and intravascular coagulation (DIC), convulsions, haemoglobinuria, impaired consciousness. ${ }^{1}$ These occur mostly with Plasmodium falciparum malaria. Occurrences of acute renal failure in severe falciparum malaria is quite common in Southeast Asia. ${ }^{2}$ and Indian subcontinent. ${ }^{3}$ Renal failure is a serious complication of malaria with a reported total mortality of $14 \%$ to $33 \% .4,5,6$ 
In view of the significant morbidity and mortality due to acute renal failure in malaria, there is an acute need to identify patients at an early stage and to intensify care given to these patients so that the burden of morbidity and mortality is reduced.

This study is undertaken to evaluate the clinical profile and outcome of acute renal failure in malaria, admitted in our institute from January 2012 to December 2012.

\section{MATERIAL AND METHODS}

This study was conducted at a Tertiary Care Hospital over a period of 12 months.

\section{AIMS AND OBJECTIVES}

Treatment often involves use of broad spectrum antibiotics. This is potentially lifesaving in severe pneumonia, but may result in development of antibiotic resistance and antibiotic induced diarrhoea and the isolation of organisms \& its sensitivity pattern is sometimes of great help, particularly to reduce the treatment time, cost of treatment. Knowing the local susceptibility profile is important, as antimicrobial therapies for LRTIs are frequently empirical and presumptive. ${ }^{2}$ Current knowledge of the organisms that cause LRTIs and their antibiotic susceptibility profiles are therefore crucially important for ensuring evidence based management approach.

A careful survey of available reports from several sources about the preventable deaths due to communicable diseases in the State of West Bengal tends to show that LRTI is one of the two major groups (The other being gastrointestinal disorders) responsible for causing nearly $50 \%$ of the total causes of diseases and deaths in the State. Documentations are inadequate except some ad-hoc studies and reports, which are indicative but not comprehensive. Reports of admissions and deaths in Calcutta Medical College during 4 consecutive years (1990-93) revealed substantial percentage of deaths due to acute respiratory illness excluding Tuberculosis (Environmental health in West Bengal: report submitted by Centre for study of Man and Environment to GOWB, Dec 1997). Communicable disease wise report of cases and deaths in West Bengal for 2009.5 shows 12698 cases of Pneumonia (7011 males and 5687 females) comprising $1.97 \%$ of illnesses with 828 deaths ( 472 males and 356 females) amounting to $22.61 \%$ of deaths (Health on the March 2009-10).

Bacterial pneumonia is a common cause of HIVassociated morbidity and mortality. The incidence of bacterial pneumonia among HIV-infected persons is greater than that in the non-infected population. Bacterial pneumonia might be the first manifestation of underlying HIV infection and can occur at any stage of HIV disease and at any CD4+ count. Risk factors associated with an increased risk for bacterial pneumonia, include low CD4+ count, injection-drug use, and cigarette smoking. Compared with persons without HIV infection, HIV-infected persons have an increased incidence of bacteraemia accompanying pneumonia, especially if they are infected with S. pneumoniae.

Before the widespread use of primary PCP prophylaxis and ART, PCP occurred in $70 \%-80 \%$ of patients with AIDS. ${ }^{6}$ Incidence of PCP has declined substantially with widespread use of prophylaxis and ART; recent incidence among patients with AIDS in Western Europe and the United States is 2-3 cases per 100 person-years. ${ }^{7}$
The majority of cases occur among patients who are unaware of their HIV infection or are not receiving ongoing HIV care. ${ }^{8}$ or among those with advanced immunosuppression (CD $4+$ counts $<100$ cells $/ \mu \mathrm{L}){ }^{9}$

Because the clinical presentation, blood tests, or chest radio-graphs are not pathognomonic for $\mathrm{PCP}$ and the organism cannot be cultivated routinely, histopathologic demonstration of organisms in tissue, bronchoalveolar lavage fluid or induced sputum samples.10,11,12,13 are required for a definitive diagnosis.

Though international guidelines are available for treating bacterial LRTI, they have not been validated very much in the Indian scenario. Scarce local information is available regarding aetiological agents of LRTI and their antibiotic sensitivity pattern. This study may be a step in formulating antibiotic use guidelines. Pneumocystis carinii (Jiroveci) is a common pathogen in LRTI in immunocompromised patients. ${ }^{14,15}$ It is rarely documented in our country. The study has tried to document PCP as one of the causative organisms of LRTI among immuno-compromised patients. A large proportion of our patients come from various parts of West Bengal, mainly rural areas. This project will contribute to improvement of care for their respiratory illnesses of infective origin.

\section{MATERIALS AND METHODS}

Specimen: Sputum-expectorated spontaneously or induced by nebulization (collection at per criteria of Murray et al. 1975).

\section{Sample Collection, Transport and Examination}

Sputum samples were collected as per criteria of Murray et al. 1975. (Squamous Epithelial Cell (SEC) less than10/Low Power Field (LPF) and except in case of neutropenia pus cells more than 25/LPF). Sputum samples containing squamous epithelial cells more than 10/LPF was rejected. Usual methods of culture sensitivity of the pathogens and tests for ESBL production by Double Disc Synergy Test (DDST), Methicillin Resistant Staphylococcus Aureus (MRSA) detection by disc diffusion using Cefoxitin disc $(30 \mu \mathrm{g})$ were done according to CLSI guidelines.

\section{Processing in the Laboratory for Bacterial Pathogen}

Sputum was Gram stained and inoculated in Blood Agar, Chocolate Agar, MacConkey Agar media and subsequently the isolate was identified by suitable biochemical and serological tests. Anaerobic culture was done in Neomycin Blood Agar using Gas Pak method. Antibiotic sensitivity test was done in Mueller-Hinton media following Kirby-Bauer Method and CLSI guidelines. Spontaneously expectorated sputum has low sensitivity and should not be submitted to the laboratory to diagnose Pneumocystis carinii pneumonia. Diagnostic sensitivities of induced sputum is between $50 \%$ and $90 \%$. (The sensitivity and specificity depend on the quality of the specimens and the experience of the microbiologist or pathologist). However, diagnostic sensitivities of sputum obtained by bronchoscopy with bronchio-alveolar lavage is $90 \%-99 \%$, transbronchial biopsy is $95 \%-100 \%$ and open lung biopsy is $95 \%-100 \%$. In our study, Giemsa staining method was used to detect the cyst and trophozoite forms. 


\section{Study Subjects}

Adults with community acquired LRTI with less than 2 weeks duration attending OPD or are admitted at Carmichael Hospital for Tropical Diseases, School of Tropical Medicine were included in the study.

Study period was one year (2014-15). Two hundred adult patients presenting with fever, cough with respiratory distress for less than two weeks' duration were selected, screened and finally recruited for the study following inclusion and exclusion criteria and after taking valid informed consent. Patients of both sexes more than 12 years of age with fever, cough, chest pain, breathlessness for less than two weeks duration, infants, children $(<12$ years), adults with cough, fever, dyspnoea for more than 2 weeks duration, patients undergoing Anti-tubercular treatment and those unwilling to participate in the study were excluded from the study.

\section{Study Design-Descriptive, Cross-Sectional Observational} Study Which Included Obtaining/Carrying Out

Informed consent of the patient.

Detailed medical history.

Physical examinations.

Routine investigation.

(a) Complete hemogram (b) Sputum for Gram stain, culture and sensitivity (c) Tests for diagnosis of PCP (d) Chest X-Ray PA view (e) HIV Ab testing by ELISA (f) CD 4 count.

Other investigations as deemed necessary e.g. routine urine and stool examination, serum urea and creatinine, Blood culture, fasting and post prandial blood sugar, were also carried out.

Parameters studied were clinical parameters, and included specific medical history along with findings on physical examinations of the patients, laboratory investigations including radiological findings, microbiological and pathological reports.

\section{Diseases under Study \\ Lower Respiratory Tract Infection (LRTI). ${ }^{16,17,18,19}$}

LRTI is defined as an acute illness (Present for 14 days or less), usually with cough as the main symptom, with at least one other lower respiratory tract symptom (Sputum production, dyspnoea, wheeze or chest discomfort/pain) and with no alternative explanation. LRTIs included Community-Acquired Pneumonia (CAP), Acute Bronchitis (AB), Acute Exacerbation of COPD (AECOPD), Acute Exacerbation of Bronchiectasis (AEBX), Pneumocystis carinii (Jirovecii) pneumonia (PCP) in immuno-compromised patients.

\section{Study Techniques}

Adult patients attending OPD or admitted in the hospital with history of fever, cough, and respiratory distress of less than 2 weeks' duration are selected considering their inclusion and exclusion criteria. After taking proper medical history, clinical examination was done and radiological reports were noted.

The sputum samples from these patients were then collected in sterile universal container and sent immediately to the laboratory for microbiological examination. In the laboratory, direct smear from sputum samples were prepared and gram stain performed to microscopically examine the smear and were simultaneously inoculated in Blood agar and MacConkey agar and incubated at $37^{\circ} \mathrm{C}$ for 8 hours.
The isolated microorganisms on culture were identified by colony characteristics, staining morphology and biochemical characteristics. Antibiotic susceptibility testing was then done using modified Kirby Bauer method by disc diffusion method as per the Clinical Laboratory Standards Institute (CLSI) guidelines. ${ }^{20,21,22,23,24}$

For gram negative organisms antibiotics tested were Ampicillin (AMP), Amoxicillin-Clavulanic Acid (AMC), Ceftriaxone (CTR), Cefotaxime (CTX), Ceftazidime (CAZ), Cefoxitin (CN), Cefepime (CPM), Piperacillin-Tazobactam (PT), Gentamicin (GM), Amikacin (AK), Imipenem (IMP), Meropenem (MRP), Ciprofloxacin (CIP) and TrimethoprimSulphamethoxazole (COT). For Gram positive organisms antibiotics tested were Amoxicillin-Clavulinic Acid (AMC), Ceftriaxone (CTR), Cefoxitin (CN), Vancomycin (VA), Linezolid (LZ), Gentamicin (GM), Amikacin (AK), Ciprofloxacin (CIP) and Trimethoprim-Sulphamethoxazole (COT).

\section{Ethical Statement}

Informed/written consent was taken in all cases. Approval of the Ethical committee was obtained.

\section{RESULTS}

\section{Total Enrolled Cases: 200}

Patients' distribution of the study population shows $88.5 \%$ $(177 / 200)$ of cases were from inpatient departments and $23 \%$ from outpatient departments. Among 200 patients, 142 (71\%) were males and 58 (29\%) were females with Male: Female ratio $2.5: 1 ; 134(67 \%)$ patients were from rural areas, while $66(33 \%)$ patients were from urban areas of West Bengal. Among the study population, 104 (52\%) were HIV nonreactive and 96 (48\%) were HIV reactive. HIV reactivity was more common among the urban patients. Socioeconomic status of the patients showed $31 \%$ were office workers, $21.5 \%$ were housewives, $15.5 \%$ were businessmen, $14.5 \%$ were drivers, $11.5 \%$ were field workers and $6 \%$ were students. Among them 54\% were smokers and $46 \%$ were non-smokers.

As shown in Table 1, out of the 200 patients, 11 patients showed no growth (5.5\%), 32 patients showed growth of normal commensal flora of throat (16\%), 148 patients showed growth of one bacteria (75\%), 7 patients showed growth of two bacteria (3.5\%) and 2 patients showed growth of Candida species (fungus). 1 patient showed Pneumocystis carinii on Giemsa stained smear, along with growth of S. pyogenes. Both the groups showed predominance of Gram positive bacterial infection. Gram negative bacteria had a second place in both the above groups. Out of 96 HIV reactive cases 78 cases have shown growth in sputum (81.25\%). Out of 104 HIV nonreactive cases, 79 have shown growth $(75.96 \%)$.

In the HIV non-reactive group, out of 104 patients, 6 patient showed no growth $(5.77 \%), 19$ patients showed growth of normal commensal flora of throat (18.27\%), 78 patients showed growth of one organism (75\%), 1 patient showed growth of two organism $(0.96 \%)$ and maximum number of LRTI cases were in the age group of 41-50 years (25.32\%). Only one patient showed growth of Candida. In the HIV reactive groups, out of 96 patients, 5 patients showed no growth (5.21\%), 13 patients showed growth of commensal (13.54\%), 72 patients showed growth of one organism (75\%), 6 showed growth of two organisms (6.25\%), and maximum numbers of LRTI cases were present in the age group of 31-40 years (38.46\%); 1 patient showed growth of Candida, 1 patient 
showed Pneumocystis carinii infection along with growth of S. pyogenes on culture.

Fig 1. Shows predominance of gram positive cocci (76.62\%), S. pyogenes followed by S. pneumoniae and then S. aureus in sputum samples of HIVR cases. The most common gram negative organism isolated was K. pneumoniae (14.29) followed by Pseudomonas SP and E. coli. Based on CD4 count in the HIV-R cohort (Table 2), S. aureus was found in patients with CD4 count $>350$, S. pyogenes, S. pneumoniae, K. pneumoniae, P. aeruginosa were found with any CD4 count and E. coli was found with CD 4 count $<100$.

S. aureus (MSSA) was found sensitive to Piperacillin/Tazobactam, Meropenem, Vancomycin and Linezolid. K. pneumoniae was found sensitive to Imipenem, Amikacin, Piperacillin/Tazobactam, Gentamicin, Levofloxacin, Cefepime, Polymyxin B and Colistin. P. aeruginosa was found sensitive to Piperacillin/Tazobactam, Imipenem, Gentamicin, Meropenem, Levofloxacin, Amikacin, Polymyxin B and Colistin. E. coli was found sensitive to Piperacillin/Tazobactam and Imipenem.

In HIV nonreactive patients (Fig 2) also Gram positive (81.01\%) bacteria (S. pyogenes, S. pneumoniae, S. aureus) were found in more number of cases than Gram negative (18.99\%) bacteria (K. pneumoniae, P. aeruginosa, Enterobacter Sp., E. coli, Acinetobacter sp.). K. pneumonia was found sensitive to Piperacillin/Tazobactam, Amikacin, Meropenem, Ciprofloxacin, Ceftriaxone. P. aeruginosa was found sensitive to Piperacillin/Tazobactam, Amikacin, Imipenem. The antibiotic sensitivity of the E. coli found in the study showed Extended Spectrum Beta-Lactamase producer (ESBL) pattern. S. aureus was found sensitive to Piperacillin/Tazobactam, Meropenem, Vancomycin, Gentamicin and Linezolid.

X-ray findings of HIV reactive patients showed Consolidation (27.08\%), Patchy Consolidation (12.5\%), Hyperinflated Lungs (17.71\%), Normal Findings (26.04\%), Calcification (11.46\%) and Blunt CP Angle (5.21\%).

X-ray findings of HIV nonreactive patients showed Consolidation (25.96\%), Patchy Consolidation (11.54\%), Hyperinflated Lungs (17.31\%), Normal Findings (25.96\%), Calcification (11.54\%) and Blunt CP Angle (7.69\%).

$\mathrm{X}$-ray report showed consolidation in 26 cases in HIV reactive patients and in 27 cases in HIV non-reactive patients. Induced sputum was collected from two patients having signs and symptoms of LRTI. Pneumocystis carinii was found in Giemsa stained smear in one case. The patient developed Pneumomediastinum and surgical emphysema. CT chest showed multiple cystic lesions in chest and confirmed Pneumomediastinum. Patient was treated in Critical Care Unit, recovered and discharged.

\section{DISCUSSION}

Lower respiratory tract infection includes acute and chronic bronchitis, bronchiolitis, pneumonia and pleural infections. A wide variety of pathogenic organisms depending on geographic variation can cause lower respiratory tract infections. In the management of this condition, specific antimicrobial therapy should be directed at the pathogen responsible in each individual case. Moreover, in recent years, there has been dramatic rise in antibiotic resistance among respiratory pathogens.
International guidelines available for treating bacterial LRTI have not been validated very much in the Indian scenario. Scarce local information is available regarding aetiological agents of LRTI and their antibiotic sensitivity pattern. This study is a step in formulating antibiotic use guideline. Pneumocystis carinii (Jiroveci) is a common pathogen of LRTI in immuno-compromised patients. It is rarely documented. The study tried to document Pneumocystis carinii as an aetiological agent of LRTI in immuno-compromised patients.

A large proportion of our patients came from rural areas of West Bengal. The findings of this study will contribute to improve the care for their respiratory illnesses of infective origin and standardize the antibacterial therapy in bacterial LRTI. This study reveals that, LRTI is common in all age groups, both sexes, all socioeconomic classes and equally in both HIVNR \& HIV-R groups. In contrast to other studies. ${ }^{25}$ percentages of bacterial isolates from LRTI patients were same (75\%) among the HIV-R \& HIV-NR cohorts in our study. However, according to our study polymicrobial growth was found to be higher among the HIV-R. In contrast to study done by Priyanka Paul Biswas et al. ${ }^{26}$ which showed predominance of gram negative organism (Klebsiella pneumoniae) isolated from inpatients group, our study shows predominance of gram positive organism among the admitted patients. However, predominance of gram positive cocci isolated from outdoor patients in our study corresponds with their findings.

In contrast to other studies. ${ }^{25,27}$ done from different parts of India which shows predominance of gram negative organisms among the two groups, our study showed predominance of Gram positive bacterial infection in both groups. In the HIV-R group, S. aureus was found in patients with CD4 count >350. S. pyogenes, S. pneumoniae, K. pneumoniae, P. aeruginosa were found with any CD4 count indicating the severity of virulence of these organisms in the HIVR patients. E. coli, a rare cause of LRTI in immunocompetent patients, was found with CD4 count $<100$ one E. coli isolate was found to be an ESBL. Otherwise, antibiotic sensitivity pattern was unremarkable.

Very few Indian studies have reported PCP in HIV. The reported incidence of Pneumocystis carinii is about $4 \%$ of opportunistic infections in HIV patients and three cases have been reported from Delhi. Unlike in the West, the prevalence of Pneumocystis carinii pneumonia is low or negligible in India. ${ }^{25}$ However, we could isolate one case of PCP from induced sputum of a patient among our study population.

There are several limitations in our study. First, not all patients who met the criteria for inclusion were enrolled in the study. Second, the sample collection may not be adequate. These issues are inherent in all trials enrolling patients with community acquired pneumonia. We have no reason to believe that the group of patients who were not included would have been substantially different from the group of patients that were included in the study.

Thus, this study gives an idea about the common etiological agents of community acquired LRTI in different groups (Age, Sex, HIV/Non-HIV) of patients and Standardization of antibacterial therapy in bacterial LRTI, as early initiation of Standard antibacterial therapy may be lifesaving in most of the cases including Pneumocystis carinii pneumonia, an etiological agent of LRTI in the HIV- reactive patients. 


\section{REFERENCES}

1. Carroll KC. Laboratory diagnosis of lower respiratory tract infections: Controversy and conundrums. J Clin Microbial 2002;40(9):3115-3120.

2. Ozyilmaz E, Akan OA, Gulhan M, et al. Major bacteria of community-acquired respiratory tract infections in Turkey. J Infect Dis 2005;58(1):50-52.

3. Erling V, Jalil F, Hanson LA, et al. The impact of climate on the prevalence of respiratory tract infection in early childhood in Lahore, Pakistan. J Pub Health 1999;21(3):331-339.

4. Liebowitz LD, Slabbert M, Huisamen A. National surveillance programme on susceptibility patterns of respiratory pathogens in South Africa: Moxifloxacin compared with eight other antimicrobial agents. J Clin Path 2003;56(5):3444-3447.

5. Health on the march 2009-10. State Bureau of Health Intelligence. Directorate of Health Services, Govt of West Bengal.

6. Phair J, Munoz A, Detels R, et al. The risk of pneumocystis carinii pneumonia among men infected with human immunodeficiency virus type 1 . Multicenter AIDS Cohort Study Group. N Engl J Med 1990;322:161-5.

7. Furrer H, Egger M, Opravil M, et al. Discontinuation of primary prophylaxis against pneumocystis carinii pneumonia in HIV-1-infected adults treated with combination antiretroviral therapy. Swiss HIV Cohort Study. N Engl J Med 1999;340:1301-6.

8. Lundberg BE, Davidson AJ, Burman WJ. Epidemiology of pneumocystis carinii pneumonia in an era of effective prophylaxis: the relative contribution of non-adherence and drug failure. AIDS 2000;14:2559-66.

9. Wolff AJ, O'Donnell AE. Pulmonary manifestations of HIV infection in the era of highly active antiretroviral therapy. Chest 2001;120:1888-93.

10. Baughman RP, Dohn MN, Frame PT. The continuing utility of bronchoalveolar lavage to diagnose opportunistic infection in AIDS patients.

Am J Med 1994;97:515-22.

11. Stover DE, Zaman MB, Hajdu SI, et al. Bronchoalveolar lavage in the diagnosis of diffuse pulmonary infiltrates in the immunosuppressed host. Ann Intern Med 1984;101: $1-7$.

12. Kovacs JA, Ng VL, Masur H, et al. Diagnosis of pneumocystis carinii pneumonia: improved detection in sputum with use of monoclonal antibodies. N Engl J Med 1988;318:589-93.

13. Roger PM, Vandenbos F, Pugliese P, et al. Persistence of pneumocystis carinii after effective treatment of P. carinii pneumonia is not related to relapse or survival among patients infected with human immuno-deficiency virus. Clin Infect Dis 1998;26:509-10.

14. Saha B, Mallik S, Bhattacharya C, Pal S, Neogi D, Pal N, et al. A study on non-tubercular pulmonary manifestations of HIV infection: AIDS 2008-x VII International AIDS Conference: Abstract no. CDB0088.
15. Usha MM, Rajendran P, Thyagarajan SP, et al. (2000): Identification of pneumocystis carinii in induced sputum of AIDS patients in Chennai, India. Indian J Pathol. Microbiol.43*(3):291-6.

16. Gupta N, Chouwdhury A, Garg U, et al. 2003: Enterobacter bacteremia Journal Assoc Physician of India 51:669-72.

17. Shankar EM, Kumarasamy N, Vignesh R, Balakrishnan $P$, Solomon SS, Murugavel KG, et al. (2007). Epidemiological studies on pulmonary pathogens in HIV-positive and negative subjects with or without community-acquired pneumonia with special emphasis on Mycoplasma pneumoniae. Japanese Journal of Infectious Diseases, 60, 337-41.

18. Randhawa HS (2000): Respiratory and systemic mycosis: An overview. Indian J. Chest Diseases and Allied Sciences 42(4):207-19.

19. Society of European clinical microbiology and infectious diseases. (Volume 17, supplement 6, November 2011).

20. Monoharan A, Chatterjee S, Mathai D (2010). Evaluation of Tigecycline activity in clinical isolates among Indian medical centres, Indian Journal of Pathol Microbiology, 53:734-7.

21. Mukhopadhyay C, Bhargava A and Ayyagari A (2003). Role of mechanical ventilation and development of multidrug resistant organisms in hospital acquired pneumonia. Indian Journal of Medical Research, 118, 229-35.

22. Patwardhan RB, Dhakephalkar PK, Niphadkar KB, et al. (2008). A study on nosocomial pathogens in ICU with special reference to multiresistant Acinetobacter baumannii harbouring multiple plasmids. Indian Journal of Medical Research, 128, 178-87.

23. Sen Gupta S, Deodher L, Kapoor H, et al. (2000): Spectrum of antibiogram against pathogens related to respiratory infarction with special reference to ceftibuten. Journal of Indian Medical Association. 98(4):196-7.

24. IDSA/ATS Guidelines for CAP in Adults • CID 2007:44 (Suppl 2) • S31.

25. Shailaja VV, Pai LA, Mathur DR, et al. Prevalence of bacterial and fungal agents causing lower respiratory tract infections in patients with human immunodeficiency virus infection. Indian Journal of Medical Microbiology 2004;22(1):28-33.

26. Paul Basu Priyanka, Prabhu Tukaram. Bacterial causes of lower respiratory tract infections in patients attending central referral hospital, Gangtok with reference to antibiotic resistance pattern. Journal of Evolution of Medical and Dental Sciences. October 2013;2(42):81268135.

27. Tripathi Purti C, Dhote Kiran. Lower respiratory tract infections: Current etiological trends and antibiogram. J Pharm Biomed Sci 2014;04(03):249-255. 


\begin{tabular}{|c|c|c|c|}
\hline & HIVR & HIVNR & Total \\
\hline $\begin{array}{c}\text { No. of patients } \\
\text { without any growth }\end{array}$ & $\begin{array}{c}5 \\
(5.26 \%)\end{array}$ & $\begin{array}{c}8 \\
(8.32 \%)\end{array}$ & $\begin{array}{c}11 \\
(5.56 \%)\end{array}$ \\
\hline $\begin{array}{l}\text { No. of patients with } \\
\text { growth of } \\
\text { commensal }\end{array}$ & $\begin{array}{c}13 \\
(13.68 \%)\end{array}$ & $\begin{array}{c}19 \\
(18.45 \%)\end{array}$ & $\begin{array}{c}32 \\
(16.16 \%)\end{array}$ \\
\hline $\begin{array}{l}\text { No. of patients with } \\
\text { growth of one } \\
\text { organism }\end{array}$ & $\begin{array}{c}71 \\
(74.74 \%)\end{array}$ & $\begin{array}{c}77 \\
(74.76 \%)\end{array}$ & $\begin{array}{c}148 \\
(74.74 \%)\end{array}$ \\
\hline $\begin{array}{l}\text { No. of patients with } \\
\text { growth of two } \\
\text { organisms }\end{array}$ & $\begin{array}{c}6 \\
(6.32 \%)\end{array}$ & $\begin{array}{c}1 \\
(0.97 \%)\end{array}$ & $\begin{array}{c}7 \\
(3.54 \%)\end{array}$ \\
\hline Total & 96 & 104 & 200 \\
\hline
\end{tabular}

\begin{tabular}{|c|c|c|c|}
\hline $\begin{array}{c}\text { CD4 } \\
\text { cell/ } \mu \mathrm{l}\end{array}$ & Male & Female & Bacterial Isolate \\
\hline$\leq 50$ & 9 & 1 & $\begin{array}{l}\text { S.pyogenes, K.pneumoniae, } \\
\text { P.aeruginosa, E.coli }\end{array}$ \\
\hline $51-100$ & 16 & 0 & $\begin{array}{c}\text { S.pyogenes, S.pneumoniae, } \\
\text { K.pneumoniae, P.aeruginosa, } \\
\text { E.coli, }\end{array}$ \\
\hline $\begin{array}{l}101- \\
200\end{array}$ & 12 & 6 & $\begin{array}{l}\text { S. pyogenes, S. pneumoniae, } \\
\text { K.pneumoniae, P.aeruginosa }\end{array}$ \\
\hline $\begin{array}{l}201- \\
300\end{array}$ & 7 & 3 & $\begin{array}{l}\text { S. pyogenes, K.pneumoniae, } \\
\text { S.pneumoniae, }\end{array}$ \\
\hline $\begin{array}{l}301- \\
350\end{array}$ & 4 & 4 & $\begin{array}{c}\text { S.pyogenes, S.pneumoniae, } \\
\text { K.pneumoniae, } \\
\text { Pseudomonas sp. }\end{array}$ \\
\hline$>350$ & 12 & 3 & $\begin{array}{l}\text { S. pyogenes, S.pneumoniae, } \\
\text { P.aeruginosa, S.aureus }\end{array}$ \\
\hline Total & 60 & 17 & \\
\hline \multicolumn{4}{|c|}{$\begin{array}{l}\text { Table 2: Correlation of bacterial } \\
\text { isolates with CD4 count in HIV group }\end{array}$} \\
\hline
\end{tabular}

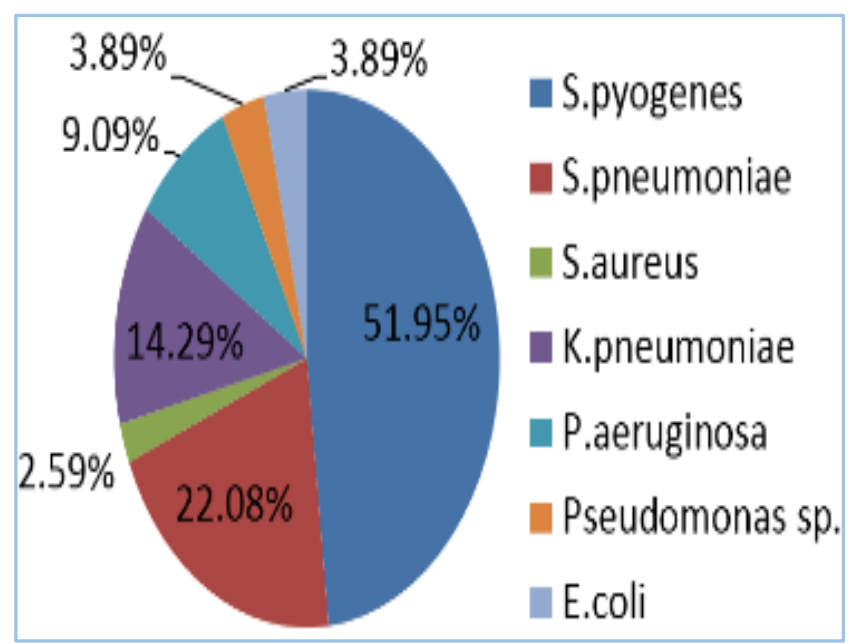

Fig. 1: No. of different bacterial Isolate in HIV Reactive population

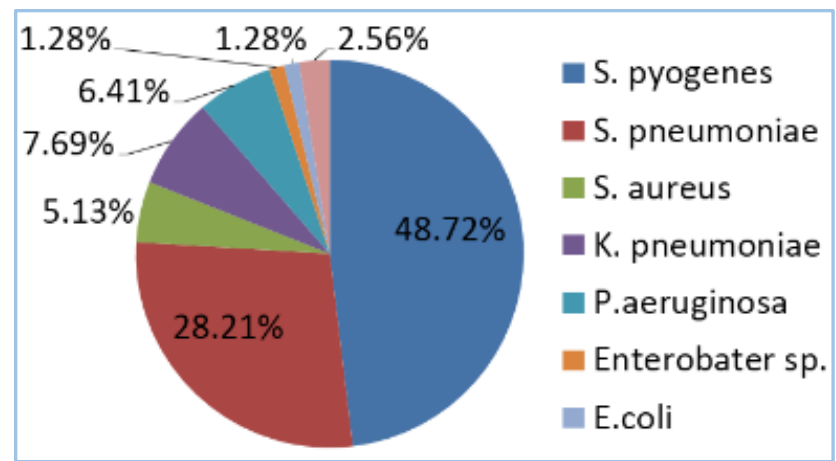

Fig. 2: No. of different bacterial isolate in HIV Nonreactive population 